\title{
A Perfectly Matched Layer Based Technique for the Scattering from 1-D Periodic Microstrip Structures
}

\author{
Dries Vande Ginste ${ }^{\# 1}$ and Hendrik Rogier \#2 \\ \# Electromagnetics Group, Department of Information Technology, Ghent University \\ Sint-Pietersnieuwstraat 41, B-9000 Gent, Belgium \\ ${ }^{1}$ dries.vandeginste@intec.uGent.be \\ ${ }^{2}$ hendrik.rogier@intec.UGent.be
}

\begin{abstract}
An efficient technique is presented to compute the scattering from one-dimensional (1-D) periodic microstrip structures, illuminated by a plane wave under perpendicular incidence. The technique relies on a Mixed Potential Integral Equation (MPIE), discretized by the Method of Moments (MoM), solving for the unknown current density flowing within a unit cell of the periodic structure. The pertinent 1-D periodic Green's functions are obtained by invoking the Perfectly Matched Layer (PML)-paradigm. The proposed formalism is illustrated and validated by evaluating the scattering from a 1-D periodic microstrip patch array.
\end{abstract}

\section{INTRODUCTION}

The study of one-dimensional (1-D) and twodimensional (2-D) periodic metallizations residing in layered background media, e.g. microstrip substrates, has been a research topic for many years [1]-[3]. The range of applications comprise antenna arrays, electromagnetic bandgap structures, frequency selective surfaces, leaky-wave antennas, high impedance metamaterial surfaces, etc.

The periodic character of these structures can be exploited by invoking the Floquet-Bloch theorem, allowing to restrict the analysis of the complete structure (with infinite extent) to one representative unit cell. Consequently, simulation methods often rely on a boundary integral equation combined with the Method of Moments (MoM) [4], solving for the unknown current density flowing within a unit cell of the periodic structure [2]. This is also the case in this contribution. At the crux of the formalism are the pertinent 1-D periodic Green's functions. Unfortunately, these Green's functions are not (immediately) available in closed form. Classically, the calculation of layered medium Green's functions necessitates the time-consuming numerical evaluation of Sommerfeld-type integrals. In [2], the 1-D periodic Green's functions in the spatial domain are obtained by means of an efficient sum of inverse Fourier transforms. Here, a different approach is adopted, based on the Perfectly Matched Layer (PML)-paradigm [5]. This paradigm allows to convert the open microstrip structure into a closed waveguide configuration, leading to closed-form series representations of the pertinent 1-D periodic Green's functions.

The outline of this paper is as follows. In Section II, the new formalism is detailed. This new technique is illustrated and validated in Section III. Conclusions are summarized in Section IV.
In the sequel, all sources and fields are assumed to be time harmonic with angular frequency $\omega$ and time dependencies $e^{j \omega t}$ are suppressed. Also, transverse to $z$ restrictions of vectors $\mathbf{v}$ are denoted $\check{\mathbf{v}} \equiv v_{x} \hat{\mathbf{x}}+v_{y} \hat{\mathbf{y}}=-\hat{\mathbf{z}} \times[\hat{\mathbf{z}} \times \mathbf{v}]$; here $\hat{\mathbf{x}}, \hat{\mathbf{y}}$, and $\hat{\mathbf{z}}$ are unit Cartesian vectors.

\section{DESCRIPTION OF THE TECHNIQUE}

A. 1-D periodic microstrip geometry

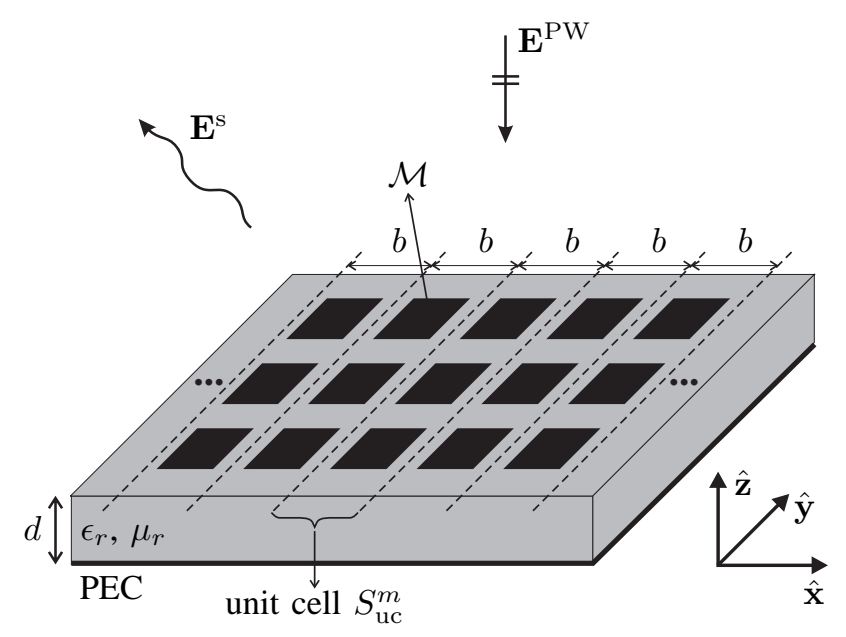

Fig. 1. 1-D periodic microstrip structure, illuminated by a plane wave $\mathbf{E}^{\mathrm{PW}}$ under perpendicular incidence, inducing a scattered field $\mathbf{E}^{\mathrm{s}}$.

Consider the 1-D periodic microstrip structure of Fig. 1. This structure consists of a dielectric layer with thickness $d$, relative permittivity $\epsilon_{r}$, and relative permeability $\mu_{r}$, placed on a perfect electrically conducting (PEC) ground plane. A 1-D periodic PEC metallization $\mathcal{M}$ resides at the substrate-air interface $z=d$. This metallization $\mathcal{M}$ is periodic along the $x$ direction and the period is $b$. In the example of Fig. 1, one unit cell consists of three square patches. A plane wave $\mathbf{E}^{\mathrm{PW}}(\mathbf{r} \equiv$ $x \hat{\mathbf{x}}+y \hat{\mathbf{y}}+z \hat{\mathbf{z}})=\mathbf{E}_{0} e^{j k_{0} z}$, with wavenumber $k_{0}=\omega / c$ and where $c$ is the speed of light, impinges perpendicularly upon the substrate. This plane wave causes an incident field $\mathbf{E}^{\mathrm{i}}(\mathbf{r})$, which in turn induces unknown current densities $\check{\mathbf{J}}(\boldsymbol{\rho} \equiv x \hat{\boldsymbol{x}}+$ $y \hat{\boldsymbol{y}}+d \hat{\boldsymbol{z}})$ flowing on $\mathcal{M}$. In response, a scattered field $\mathbf{E}^{\mathrm{S}}(\mathbf{r})$ is generated. The total electric field $\mathbf{E}^{\mathrm{t}}(\mathbf{r})$ comprises both the incident and the scattered fields, viz. $\mathbf{E}^{\mathrm{t}}(\mathbf{r})=\mathbf{E}^{\mathrm{i}}(\mathbf{r})+\mathbf{E}^{\mathrm{S}}(\mathbf{r})$. 


\section{B. Construction of the MPIE-MoM}

Upon knowledge of the pertinent 1-D periodic layered medium Green's function $G_{A}^{\text {per }}\left(\boldsymbol{\rho} \mid \boldsymbol{\rho}^{\prime}\right)$ for the magnetic vector potential and $G_{V}^{\text {per }}\left(\boldsymbol{\rho} \mid \boldsymbol{\rho}^{\prime}\right)$ for the electric scalar potential, a Mixed Potential Integral Equation (MPIE) is constructed by demanding that the total electric field tangential to the metallization $\mathcal{M}$ vanishes:

$$
\begin{aligned}
-\check{\mathbf{E}}^{\mathrm{i}}(\boldsymbol{\rho})= & \check{\mathbf{E}}^{\mathrm{s}, \mathrm{r}}(\boldsymbol{\rho}) \\
\equiv & -j \omega \iint_{\mathcal{M}^{m}} G_{A}^{\mathrm{per}}\left(\boldsymbol{\rho} \mid \boldsymbol{\rho}^{\prime}\right) \check{\mathbf{J}}\left(\boldsymbol{\rho}^{\prime}\right) d \boldsymbol{\rho}^{\prime} \\
& +\frac{1}{j \omega} \check{\nabla} \iint_{\mathcal{M}^{m}} G_{V}^{\mathrm{per}}\left(\boldsymbol{\rho} \mid \boldsymbol{\rho}^{\prime}\right)\left(\check{\nabla}^{\prime} \cdot \check{\mathbf{J}}\left(\boldsymbol{\rho}^{\prime}\right)\right) d \boldsymbol{\rho}^{\prime}, \\
& \forall \boldsymbol{\rho} \in \mathcal{M}^{m}
\end{aligned}
$$

with $\check{\nabla}=\frac{\partial}{\partial x} \hat{\mathbf{x}}+\frac{\partial}{\partial y} \hat{\mathbf{y}}$ and where $\mathcal{M}^{m}$ is that part of the metallization that lies within one unit cell $S_{\mathrm{uc}}^{m}=$ $\{\boldsymbol{\rho} \equiv x \hat{\boldsymbol{x}}+y \hat{\boldsymbol{y}}+d \hat{\boldsymbol{z}}: m b \leq x<(m+1) b,-\infty<y<\infty\}$, $m \in \mathbb{Z}$. The integral equation (1) can be solved by the MoM. Thereto, the metallization $\mathcal{M}^{m}$ is approximated by a (potentially nonuniform) rectilinear mesh with $N$ interior edges. Next, $\breve{\mathbf{J}}(\boldsymbol{\rho})$ is expanded into a set of vector rooftop basis functions [6] $\check{\mathbf{w}}_{j}(\boldsymbol{\rho})=w_{x, j}(\boldsymbol{\rho}) \hat{\mathbf{x}}+w_{y, j}(\boldsymbol{\rho}) \hat{\mathbf{y}}, j=1, \ldots, N$, with support $\mathcal{M}_{j}^{m} \subset \mathcal{M}^{m}$ comprising two patches that are joined by the mesh's $j^{\text {th }}$ interior edge:

$$
\check{\mathbf{J}}(\boldsymbol{\rho})=\sum_{j=1}^{N} I_{j} \check{\mathbf{w}}_{j}(\boldsymbol{\rho}) .
$$

Inserting (2) into (1) and applying a Galerkin testing procedure yields an $N \times N$ linear system

$$
\mathbf{V}=\overline{\mathbf{Z}} \cdot \mathbf{I} \text {. }
$$

In (3), the $N$-vector I contains the unknown expansion coefficients $I_{j}, j=1, \ldots, N$, and the elements of the $N$-vector $\mathbf{V}$ and the $N \times N$ matrix $\overline{\mathbf{Z}}$ are given by

$$
\begin{aligned}
V_{i}= & \iint_{\mathcal{M}_{i}^{m}} \check{\mathbf{E}}^{\mathrm{i}}(\boldsymbol{\rho}) \cdot \check{\mathbf{w}}_{i}(\boldsymbol{\rho}) d \boldsymbol{\rho} \\
Z_{i j}= & j \omega \iint_{\mathcal{M}_{i}^{m}} \iint_{\mathcal{M}_{j}^{m}} G_{A}^{\mathrm{per}}\left(\boldsymbol{\rho} \mid \boldsymbol{\rho}^{\prime}\right)\left(\check{\mathbf{w}}_{i}(\boldsymbol{\rho}) \cdot \check{\mathbf{w}}_{j}\left(\boldsymbol{\rho}^{\prime}\right)\right) d \boldsymbol{\rho}^{\prime} d \boldsymbol{\rho} \\
& +\frac{1}{j \omega} \iint_{\mathcal{M}_{i}^{m}} \iint_{\mathcal{M}_{j}^{m}} G_{V}^{\mathrm{per}}\left(\boldsymbol{\rho} \mid \boldsymbol{\rho}^{\prime}\right)\left[\left(\check{\boldsymbol{\nabla}} \cdot \check{\mathbf{w}}_{i}(\boldsymbol{\rho})\right)\right. \\
& \left.\left(\check{\boldsymbol{\nabla}}^{\prime} \cdot \check{\mathbf{w}}_{j}\left(\boldsymbol{\rho}^{\prime}\right)\right)\right] d \boldsymbol{\rho}^{\prime} d \boldsymbol{\rho}
\end{aligned}
$$

The linear system (3) is solved using direct or iterative solution schemes.

\section{PML-based 1-D periodic Green's functions}

As stated before, the MPIE-MoM formalism requires the knowledge of $G_{A}^{\text {per }}\left(\boldsymbol{\rho} \mid \boldsymbol{\rho}^{\prime}\right)$ and $G_{V}^{\mathrm{per}}\left(\boldsymbol{\rho} \mid \boldsymbol{\rho}^{\prime}\right)$. These 1-D periodic Green's functions can be determined through application of the PML-paradigm, described in [5], [7]. To this end, the semi-infinite layer of air $z>d$ is closed by a PEC-backed PML, which corresponds to placing a PEC-plate at a complex distance $z=d+\mathcal{D}$ above the microstrip configuration

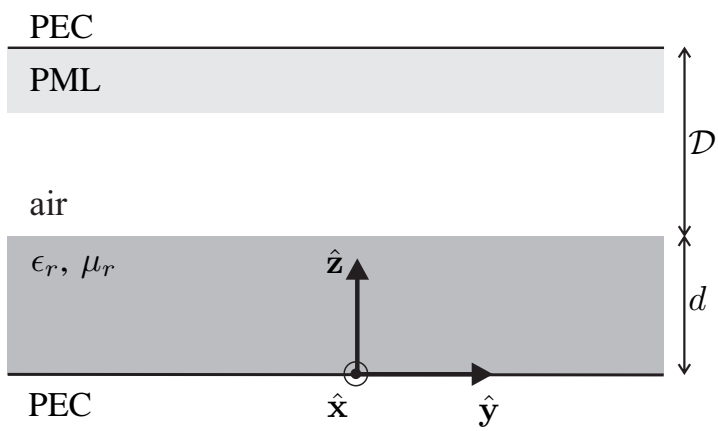

Fig. 2. Frontal view of a PEC-backed PML-closed microstrip substrate.

(Fig. 2). The complex value of $\mathcal{D}$ must be chosen such that the PML provides damping along the direction perpendicular to the layer interfaces, i.e. the direction $z$. This damping is achieved when the real part $\Re(\mathcal{D})>0$ and the imaginary part $\Im(\mathcal{D})<0$. Due to the reflectionless absorption of waves impinging upon the PML, the PEC-backed PML-closed microstrip structure closely mimics the behavior of the original open structure. However, whereas the open structure's modal spectrum contains a continuous set of radiation modes, necessitating the numerical evaluation of Sommerfeld-integrals, the closed structure only exhibits discrete sets of eigenmodes. Consequently, the Green's functions can be written as closedform series expansions:

$$
\begin{aligned}
G_{A}^{\mathrm{per}}\left(\boldsymbol{\rho} \mid \boldsymbol{\rho}^{\prime}\right)= & -\frac{j}{2} \sum_{n=1}^{\infty} \sum_{m=-\infty}^{+\infty} \frac{H_{0}^{(2)}\left(\beta_{\mathrm{TE}, n} \Delta_{m}\right)}{M^{\mathrm{TE}}\left(\beta_{\mathrm{TE}, n}\right)}, \\
G_{V}^{\mathrm{per}}\left(\boldsymbol{\rho} \mid \boldsymbol{\rho}^{\prime}\right)= & -\frac{j \omega^{2}}{2} \sum_{n=1}^{\infty} \sum_{m=-\infty}^{+\infty} \frac{H_{0}^{(2)}\left(\beta_{\mathrm{TE}, n} \Delta_{m}\right)}{\beta_{\mathrm{TE}, n}^{2} M^{\mathrm{TE}}\left(\beta_{\mathrm{TE}, n}\right)} \\
& +\frac{j}{2} \sum_{n=1}^{\infty} \sum_{m=-\infty}^{+\infty} \frac{H_{0}^{(2)}\left(\beta_{\mathrm{TM}, n} \Delta_{m}\right)}{\beta_{\mathrm{TM}, n}^{2} M^{\mathrm{TM}}\left(\beta_{\mathrm{TM}, n}\right)} .
\end{aligned}
$$

Here, $\Delta_{m}=\sqrt{\left(x-x^{\prime}-m b\right)^{2}+\left(y-y^{\prime}\right)^{2}}, H_{0}^{(2)}(\cdot)$ is the zeroth-order Hankel function of the second kind, and

$$
\begin{aligned}
M^{\mathrm{TE}}(\beta)= & \frac{d}{\mu_{1}} \frac{1}{\sin ^{2} \gamma_{1} d}-\frac{\cot \gamma_{1} d}{\mu_{1} \gamma_{1}} \\
& +\frac{\mathcal{D}}{\mu_{0}} \frac{1}{\sin ^{2} \gamma_{0} \mathcal{D}}-\frac{\cot \gamma_{0} \mathcal{D}}{\mu_{0} \gamma_{0}}, \\
M^{\mathrm{TM}}(\beta)= & \frac{\epsilon_{1} \cot \gamma_{1} d}{\gamma_{1}^{3}}+\frac{\epsilon_{1} d}{\gamma_{1}^{2} \sin ^{2} \gamma_{1} d} \\
& +\frac{\epsilon_{0} \cot \gamma_{0} \mathcal{D}}{\gamma_{0}^{3}}+\frac{\epsilon_{0} \mathcal{D}}{\gamma_{0}^{2} \sin ^{2} \gamma_{0} \mathcal{D}},
\end{aligned}
$$

with $\gamma_{0}=\sqrt{k_{0}^{2}-\beta^{2}}$ and $\gamma_{1}=\sqrt{k_{0}^{2} \epsilon_{r} \mu_{r}-\beta^{2}} \cdot \beta_{\mathrm{TE}, n}$ and $\beta_{\mathrm{TM}, n}$ represent the propagation constants of the Transverse Electric (TE) and the Transverse Magnetic (TM) polarized eigenmodes of the PEC-backed PML-closed waveguide. All these propagation constants have a negative imaginary part, and hence, only a limited set of modes needs to be retained in the summations. Via the Poisson summation, (6) and (7) are 


$$
\begin{aligned}
& G_{A}^{\mathrm{per}}\left(\boldsymbol{\rho} \mid \boldsymbol{\rho}^{\prime}\right) \\
& =-\frac{j}{b} \sum_{n=1}^{\infty} \sum_{m=-\infty}^{+\infty} \frac{e^{-j \frac{2 \pi m}{b}\left(x-x^{\prime}\right)} e^{-j \sqrt{\beta_{\mathrm{TE}, n}^{2}-\left(\frac{2 \pi m}{b}\right)^{2}}\left|y-y^{\prime}\right|}}{M^{\mathrm{TE}}\left(\beta_{\mathrm{TE}, n}\right) \sqrt{\beta_{\mathrm{TE}, n}^{2}-\left(\frac{2 \pi m}{b}\right)^{2}}} \\
& G_{V}^{\mathrm{per}}\left(\boldsymbol{\rho} \mid \boldsymbol{\rho}^{\prime}\right) \\
& =-\frac{j \omega^{2}}{b} \sum_{n=1}^{\infty} \sum_{m=-\infty}^{+\infty} \frac{e^{-j \frac{2 \pi m}{b}\left(x-x^{\prime}\right)} e^{-j \sqrt{\beta_{\mathrm{TE}, n}^{2}-\left(\frac{2 \pi m}{b}\right)^{2}}\left|y-y^{\prime}\right|}}{M^{\mathrm{TE}}\left(\beta_{\mathrm{TE}, n}\right) \sqrt{\beta_{\mathrm{TE}, n}^{2}-\left(\frac{2 \pi m}{b}\right)^{2}}} \\
& \quad+\frac{j}{b} \sum_{n=1}^{\infty} \sum_{m=-\infty}^{+\infty} \frac{e^{-j \frac{2 \pi m}{b}\left(x-x^{\prime}\right)} e^{-j \sqrt{\beta_{\mathrm{TM}, n}^{2}-\left(\frac{2 \pi m}{b}\right)^{2}}\left|y-y^{\prime}\right|}}{\beta_{\mathrm{TM}, n}^{2} M^{\mathrm{TM}}\left(\beta_{\mathrm{TM}, n}\right) \sqrt{\beta_{\mathrm{TM}, n}^{2}-\left(\frac{2 \pi m}{b}\right)^{2}}}
\end{aligned}
$$

Both kinds of series are useful. On the one hand, series (6) and (7) are required to calculate interactions $Z_{i j}$ (5) whenever $\left|y-y^{\prime}\right|$ is small. On the other hand, series (10) and (11) converge very rapidly for large enough $\left|y-y^{\prime}\right|$ and this property is exploited in the implementation of the new 1-D periodic MPIE-MoM. For $m=0$, the series (6) and (7) reduce to the PML-based series for non-periodic microstrip substrates [8] and it is known that these series become impractical for $\Delta_{0}=\left|\boldsymbol{\rho}-\boldsymbol{\rho}^{\prime}\right| \rightarrow 0$. This issue can be easily alleviated by replacing the term $m=0$ in the summation with the classical Sommerfeld-integral based non-periodic Green's function. This yields alternative series, valid for small $\left|\boldsymbol{\rho}-\boldsymbol{\rho}^{\prime}\right|$, leading to very accurate calculations of the self-patch interactions $Z_{i i}, i=1, \ldots, N$.

\section{NUMERICAL EXAMPLE AND VALIDATION}

The above described PML-based MPIE-MoM is now illustrated and validated by considering a representative example. The results are obtained using a Linux-based 64-bit AMD Opteron 270 computer with $8 \mathrm{~GB}$ of RAM running at $2 \mathrm{GHz}$. A BiCGstab iterative solver is used to solve linear system (3).

Again consider the configuration of Fig. 1, where the microstrip substrate has a thickness $d=3.17 \mathrm{~mm}$, a relative permittivity $\epsilon_{r}=11.7$, and a relative permeability $\mu_{r}=1$. The metallization is the patch array shown in Fig. 1. Each of the three PEC patches in the unit cell have a size of $10 \mathrm{~mm} \times 10 \mathrm{~mm}$. The period is $b=40 \mathrm{~mm}$ and the spacing between the patches along the $y$-direction is $10 \mathrm{~mm}$. The structure is illuminated by a plane wave $\mathbf{E}^{\mathrm{PW}}(\mathbf{r})=e^{j k_{0} z} \hat{\mathbf{y}}$ at an angular frequency of $\omega=2 \pi 10 \mathrm{GHz}$. The current density $\breve{\mathbf{J}}(\boldsymbol{\rho})$, induced by the plane wave, is shown in Fig. 3. Obviously, as the plane wave's electric field is $y$-oriented, the $y$-component of the current density (Fig. 3(b)) is much larger than its $x$-component (Fig. 3(a)). Our approach is now validated by considering the far-field $\mathbf{E}^{\mathrm{s}, \mathrm{ff}}(\mathbf{r})$ scattered from the antenna array, expressed as

$$
\mathbf{E}^{\mathrm{s}, \mathrm{ff}}(\mathbf{r}) \approx\left(\mathcal{F}_{\theta}(\theta, \phi) \hat{\boldsymbol{\theta}}+\mathcal{F}_{\phi}(\theta, \phi) \hat{\boldsymbol{\phi}}\right) \frac{e^{-j k_{0} R}}{R}
$$

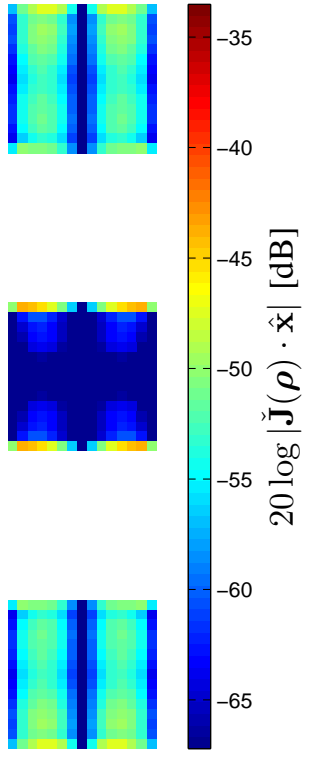

(a) $x$-oriented current density

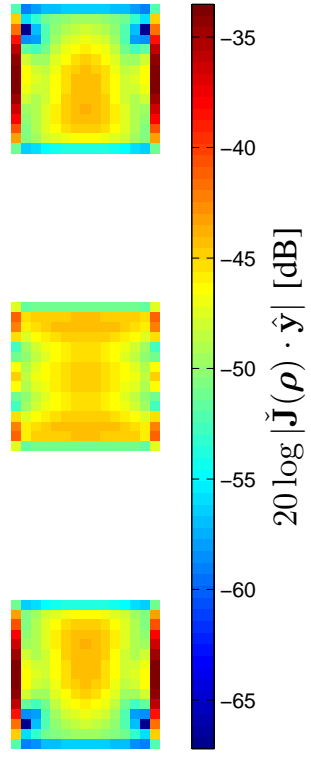

(b) $y$-oriented current density
Fig. 3. Current density within one unit cell of the patch array.

In (12) the usual spherical coordinate system is $\quad$ used: $\quad x=R \sin \theta \cos \phi, \quad y=R \sin \theta \sin \phi$, $z=R \cos \theta, \quad \hat{\boldsymbol{\theta}}=\cos \theta \cos \phi \hat{\mathbf{x}}+\cos \theta \sin \phi \hat{\mathbf{y}}-\sin \theta \hat{\mathbf{z}}$, and $\hat{\boldsymbol{\phi}}=-\sin \phi \hat{\mathbf{x}}+\cos \phi \hat{\mathbf{y}}$. The far-field pattern's components $\mathcal{F}_{\theta}(\theta, \phi)$ and $\mathcal{F}_{\phi}(\theta, \phi)$ are calculated based on the current density on the metallization using the stationary phase method [9]. After solving the periodic problem, to calculate the scattering pattern, here, we only consider seven unit cells, with seven identical current densities as shown in Fig. 3, spaced $b=40 \mathrm{~mm}$ apart from each other. The far-field, scattered from these seven unit cells, is shown in Fig. 4. The results are compared with the solution of a reference program, the SVD-PML-MLFMA [10]. This is a tool developed for the simulation of finite, non-periodic structures. With the SVD-PML-MLFMA a finite array of $7 \times 3$ patches is simulated. Although only seven periods are used, good agreement is already observed, as such validating the approach described in this contribution. In Fig. 4(a), apart from the specular reflection at $\theta_{\text {spec }}=0^{\circ}$, two grating lobes at angles $\theta_{\mathrm{gr}, 1}=\arcsin \frac{2 \pi}{b k_{0}}=48.5^{\circ}$ and $\theta_{\mathrm{gr}, 2}=\arcsin \frac{-2 \pi}{b k_{0}}=-48.5^{\circ}$ are observed. In the $x z$-plane (Fig. 4(a)), the $\theta$-component of the scattered far-field is very small. In the $y z$-plane (Fig. 4(b)), there is no significant $\phi$-component.

Notwithstanding the fact that the SVD-PML-MLFMA is a MoM-based simulation tool that is hugely accelerated by means of a multilevel fast multipole technique, the 1-D periodic MPIE-MoM approach is still faster. Both solvers are set to reach 6 digits of accuracy (leading to the results presented in Fig. 4). The total solution time using the 1-D periodic MPIEMoM was $42.60 \mathrm{sec}$. The solution time of the SVD-PML- 
